\title{
Simulation and Analysis of MC-CDMA System under AWGN and Rayleigh Fading Channel
}

1st Bhavana kulhare, 2nd Dr. Poonam sinha

Dept. of Electronics \& communication, BUIT,BU Barkatullah University,

Bhopal(M.P),India

bhawnakulhare@gmail.com

Dept. of Electronics \& communication, BUIT,BU, Barkatullah University, Bhopal,

Bhopal(M.P),India

poonamuit@yahoo.com

\section{ABSTRACT}

This paper presents a detailed analysis of Multicarrier Code Division Multiple Access (MC-CDMA) system over Additive White Gaussian Noise (AWGN) and Rayleigh Fading Channel with analysisof the effects of different number of subcarriers and multiuser scenarios and at the end a comparison with standard technique is also performed. The complete analysis is performed by simulating the mathematical model of the MC-CDMA system with various combinations of subcarriers, number of users, SNR, path gains and path delays etc. the simulation results shows that the MC-CDMA is an effective way to overcome the multipath fading losses and spectrum utilization with relatively simple system architecture.

\section{Keywords}

Multicarrier Code Division Multiple Access (MC-CDMA), Rayleigh Fading Channel, Additive White Gaussian Noise (AWGN).

\section{Council for Innovative Research}

Peer Review Research Publishing System

Journal: INTERNATIONAL JOURNAL OF COMPUTERS \& TECHNOLOGY

Vol 6, No 2

editor@cirworld.com

www.cirworld.com, member.cirworld.com 


\section{INTRODUCTION}

Multi-Carrier Code Division Multiple Access (MC-CDMA) is a multiple access scheme used in OFDM-based telecommunication systems, allowing the system to support multiple users at the same time.

MC-CDMA spreads each user symbol in the frequency domain. That is, each user symbol is carried over multiple parallel subcarriers, but it is phase shifted (typically 0 or 180 degrees) according to a code value. The code values differ per subcarrier and per user. The receiver combines all subcarrier signals, by weighing These to compensate varying signal strengths and undo the code shift. The receiver can separate signals of different users, because these have different (e.g. orthogonal) code values [1].Since each data symbol occupies a much wider bandwidth (in hertz) than the data rate (in $\mathrm{bit} / \mathrm{s}$ ), a signal-to-noise-plus-interference ratio (if defined as signal power divided by total noise plus interference power in the entire transmission band) of less than $0 \mathrm{~dB}$ is feasible.One way of interpreting MC-CDMA is to regard it as a directsequence CDMA signal (DS-CDMA) which is transmitted after it has been fed through an inverse FFT (Fast Fourier Transform).

A number of alternative possibilities exist as to how this frequency domain spreading can take place, such as by using a long PN code and multiplying each data symbol. Once frequency domain spreading has taken place and the OFDM subcarriers have all been allocated values, OFDM modulation then takes place using the IFFT to produce an OFDM symbol; the OFDM guard interval is then added; and if transmission is in the downlink direction each of these resulting symbols are added together prior to transmission. The rest of paper is arranged as the second section presents a brief review of the relevant work done so far then section third and four explains concepts of MC-CDMA system and channel properties for AWGN and Rayleigh fading channel followed by simulation results and conclusion in fifth and sixth section.

\section{Literature Review}

This section presents a brief introduction and abstracts of the relevant work which are found useful during the designing and simulating of proposed model. Xue Li, Ruolin Zhou, Steven Hong and Zhiqiang Wu [2] presented a novel ICl cancellation schemethat can eliminate the $\mathrm{ICl}$ entirely and offer a MC-CDMA mobilesystem with the same BER performance of a MC-CDMAsystemwithout ICI. More importantly, theproposed ICI cancellationscheme (namely TotallCI Cancellation) does not lower thetransmission rate or reduce the bandwidthefficiency. Specifically,by exploiting frequency offset quantization, the proposed schemetakes advantage of the orthogonality of the $\mathrm{ICl}$ matrix and offersperfect $\mathrm{ICl}$ cancellation and significant BER improvement at linearly growing cost. Simulation results in AWGN channel andmulti-path fading channel confirm the excellent performance ofthe proposed Total ICl Cancellation scheme in the presence of frequency offset or time variations in the channel, outperforming existing $\mathrm{ICl}$ cancellation methods. B. Sarala and D.S.Venkateswarulu [3] presented a new technique for reduction in PAPR of the Multicarrier Code Division Multiple Access (MC CDMA) signals based on combining the Discrete Transform either Discrete Cosine Transform (DCT) or multi-resolution Discrete Wavelet Transform (DWT) with companding is proposed.MC-CDMA Based IEEE 802.11 Wireless LAN is evaluated by Georgios Orfanos, Jorg Habethaand Ling Liu [4]. They proposed and evaluated the modified version of the IEEE 802.11a protocol. They combine Multi-Carrier Code Division Multiple Access (MC-CDMA), a novel, high capacity multicarrier modulation technique, with the standard Medium Access Control (MAC) protocol of the 802.11 Wireless Local Area Network (WLAN). The suggested system utilizes spread spectrum to divide the channel bandwidth in parallel code channels, and allows for a number of mobile terminals to share the medium in a more fair and efficient way. The proposed system has been evaluated using a protocol simulator, MACNET-2, and the performance results are discussed in their paper. Performance Analysis of Coded MC-CDMA in Powerline Communication Channel with Impulsive Noise is presented by S.M.Navidpour, P. Amirshahi and M. Kavehrad (FIEEE) [5]. In their work the bit error rate (BER) performance of the MC-CDMA system under impulsive noise and frequency fading is theoretically analyzed and closed form expression for this performance is derived. Furthermore, a theoretical upper bound for performance of coded MC-CDMA system is derived, given perfect interleaving and effect of interleave length on coding performance is also studied. Simulations show that the upper bound is quite tight for the case of employing a longer interleave. Kattoush A. H. and Qasaymeh M. M. presented Design and Simulation of MC-CDMA Transceiver via Slantlet Transform [6]. In their work, a novel SLT-MC-CDMA transceiver design is presented based on the SLT-OFDM that is used as a basic building block in the design of MC-CDMA transceiver to maintain the orthogonality against the multipath frequency Selective Fading Channels (SFC). Simulation results are provided to demonstrate the significant gain in the performance of the proposed technique. The Bit Error Rate (BER) of SLT-MC-CDMA scheme is compared with FFT-MC-CDMA and tested in Additive White Gaussian Noise (AWGN), Flat Fading and Selective Fading Channels (SFH). The simulation results confirmed that the proposed system outperforms the reference one.

\section{Multicarrier Code Division Multiple Access (MC-CDMA)}

In MC-DS-CDMA systems, the original data stream is first converted from serial to parallel, spread by using the user specific spreading code in the time domain and finally, each of these data streams modulate a subcarrier. In that sense, each frequency band is used to transmit a narrowband direct sequence signal. As expected, MC-CD-CDMA is computationally more expensive than DS-CDMA. 


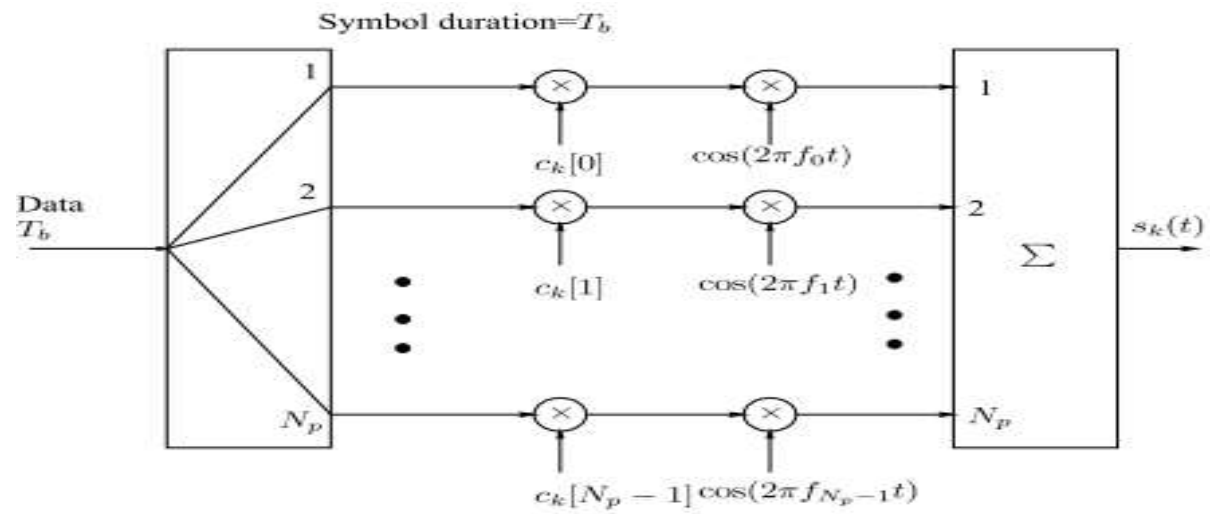

Figure 1: The transmitter diagram of frequency-domain spreading assisted MC-CDMA systems.

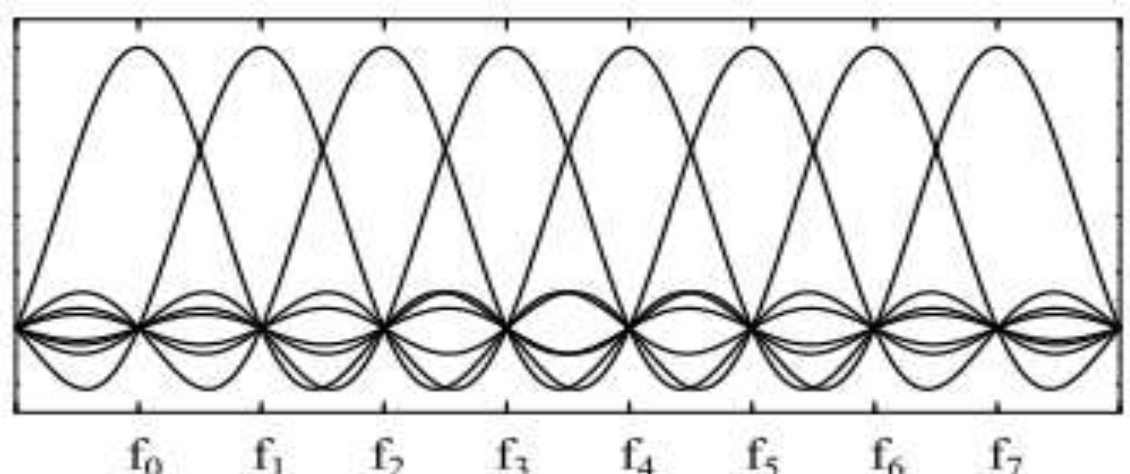

$\begin{array}{llllllll}\mathrm{f}_{0} & \mathrm{f}_{1} & \mathrm{f}_{2} & \mathrm{f}_{3} & \mathrm{f}_{4} & \mathrm{f}_{5} & \mathrm{f}_{6} & \mathrm{f}_{7}\end{array}$

Figure 2: Spectrum of the frequency-domain spreading assisted MC-CDMA signal.

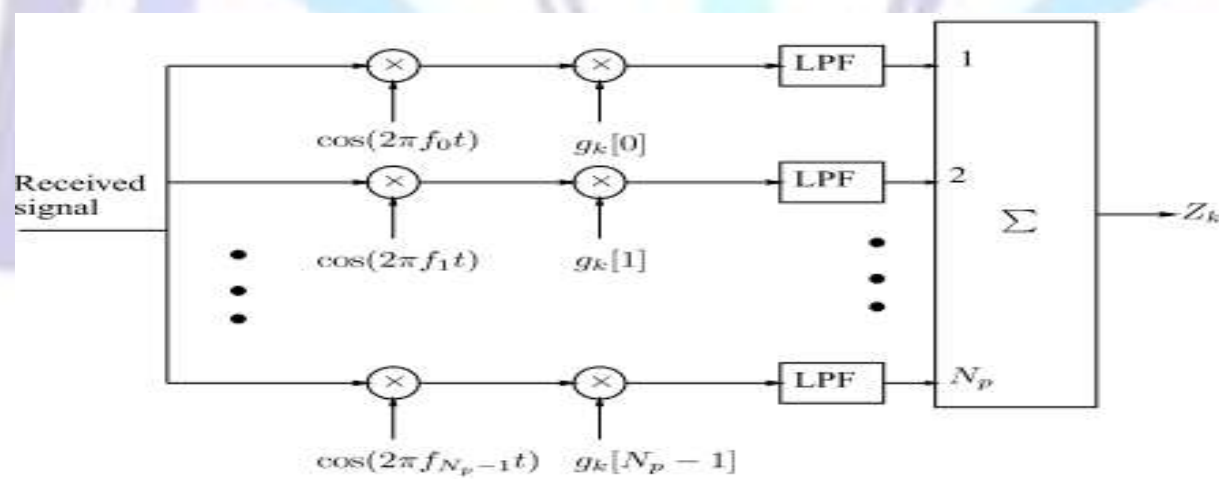

Figure 3: The receiver block diagram of frequency-domain spreading assisted MC-CDMA systems.

Fig. 1, 2, 3 shows the block diagram of the MC-CDMA system. At the transmitter, each user's modulated signal is spread by a pre-assigned spreading code. The frequency domain spread signal is interleaved and then converted into time domain by IFFT. Here the interleaving operation is used to map the chips of each symbol onto equally-spaced subcarriers. At the receiver, after removing the $\mathrm{CP}$, the time domain signal is converted into frequency domain by FFT and a frequency domain MMSE equalizer is implemented to recover the orthogonality of the spreading codes. Then the equalized signal is despread directly to obtain the desired user's signal. 


\section{Channel Models}

In telecommunications and computer networking, a communication channel, or channel, refers either to a physical transmission medium such as a wire or to a logical connection over a multiplexed medium such as a radio channel. A channel is used to convey an information signal, for example a digital bit stream, from one or several senders (or transmitters) to one or several receivers. A channel has a certain capacity for transmitting information, often measured by its bandwidth in $\mathrm{Hz}$ or its data rate in bits per second.

\subsection{AWGN Channel:}

Additive white Gaussian noise (AWGN) is a channel model in which the only impairment to communication is a linear addition of wideband or white noise with a constant spectral density (expressed as watts per hertz of bandwidth) and a Gaussian distribution of amplitude. The model does not account for fading, frequency selectivity, interference, nonlinearity or dispersion. However, it produces simple and tractable mathematical models which are useful for gaining insight into the underlying behavior of a system before these other phenomena are considered.

\subsection{Fading Channels:}

In wireless communications, fading is deviation of the attenuation affecting a signal over certain propagation media. The fading may vary with time, geographical position or radio frequency, and is often modeled as a random process. A fading channel is a communication channel comprising fading. In wireless systems, fading may either be due to multipath propagation, referred to as multipath induced fading, or due to shadowing from obstacles affecting the wave propagation, sometimes referred to as shadow fading.

\section{Simulated Results:}

The following simulation parameters are set for the simulation of the system

Number of Bits: 10000

Number of Users: 2, 4, 15

Number of Sub-Carriers: 16

AWGN Channel Parameters

SNR (minimum): $0 \mathrm{~dB}$

SNR (maximum): $20 \mathrm{~dB}$

Rayleigh Fading Channel Parameters

Number of Paths: 4

Gains of Path: 0.6, 0.2, 0.1, 0.1.

Delays of Path: $0,1,2,3$

All results are calculated for 103 bits of transmission, the length of spreading code is same as number of sub-carriers and results are 


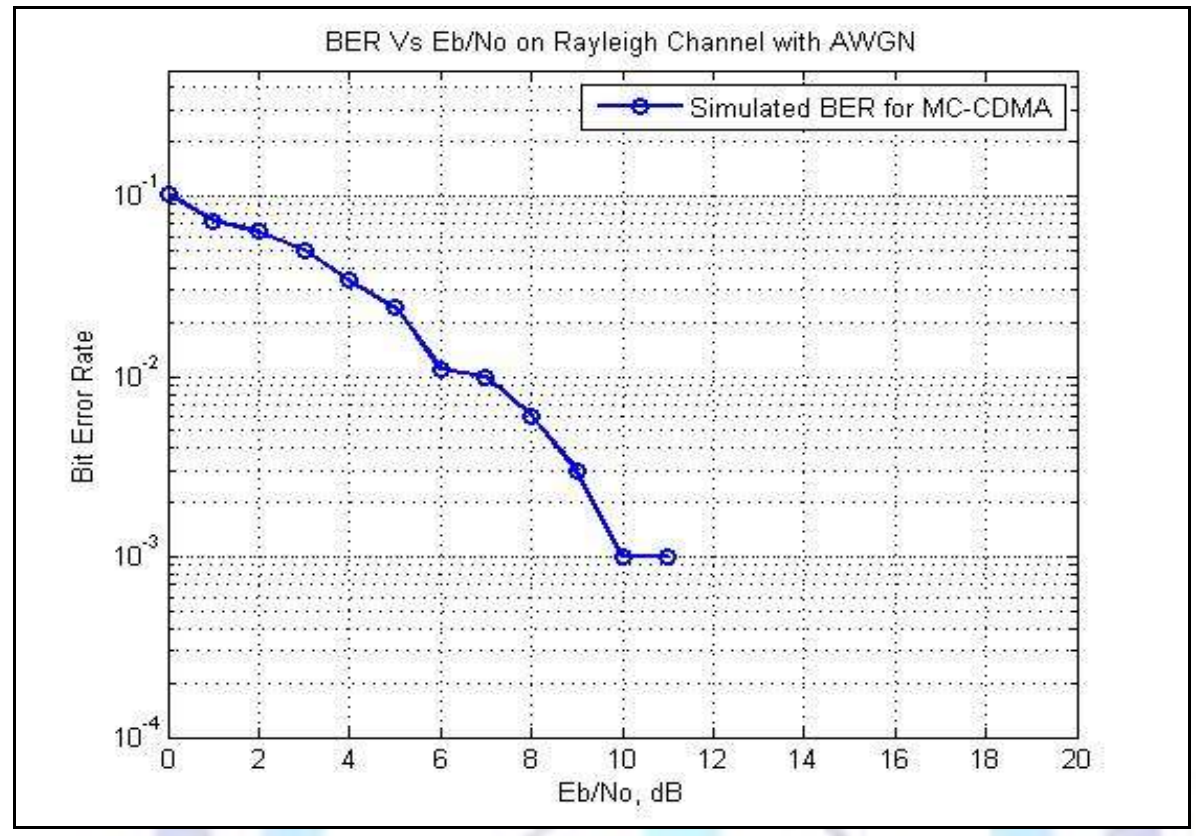

Figure 4: SNR/BER for 2 users16 sub-carriers.

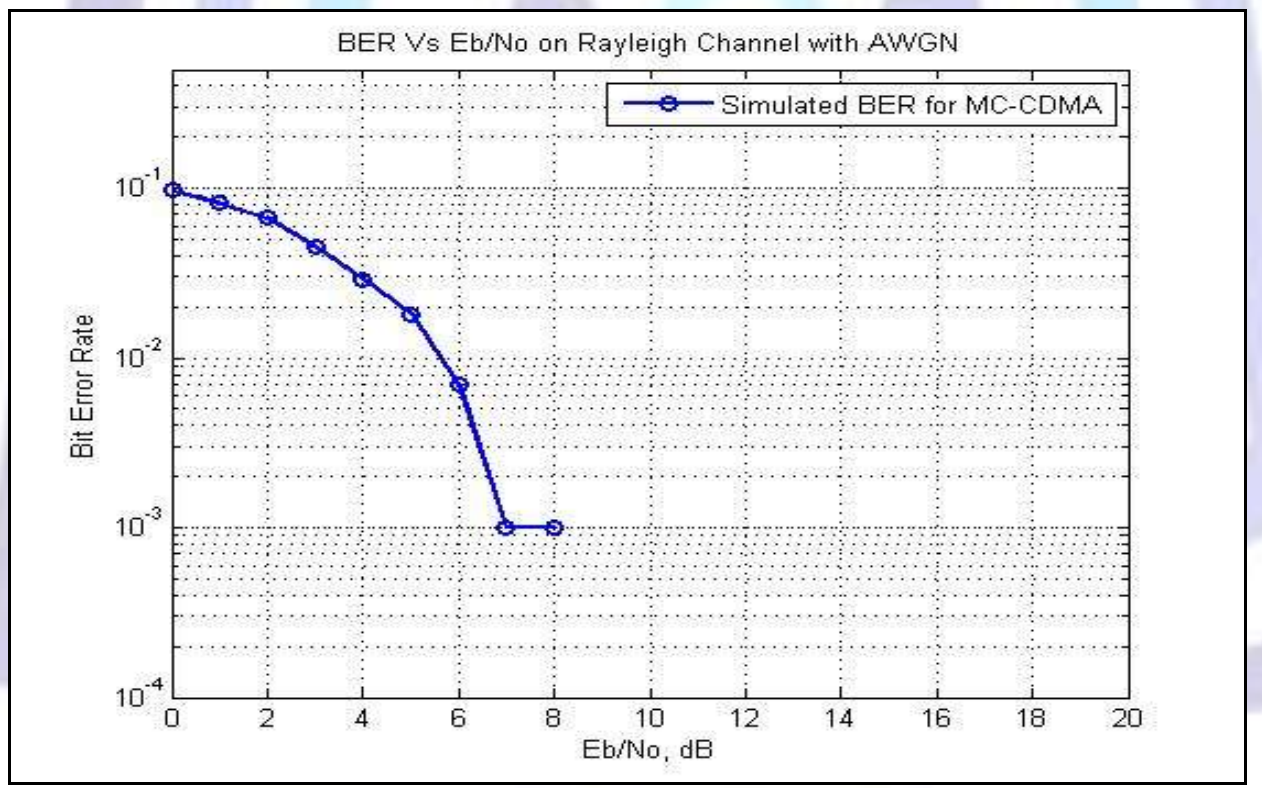

Figure 5: SNR/BER for 4 users 16 sub-carriers. 


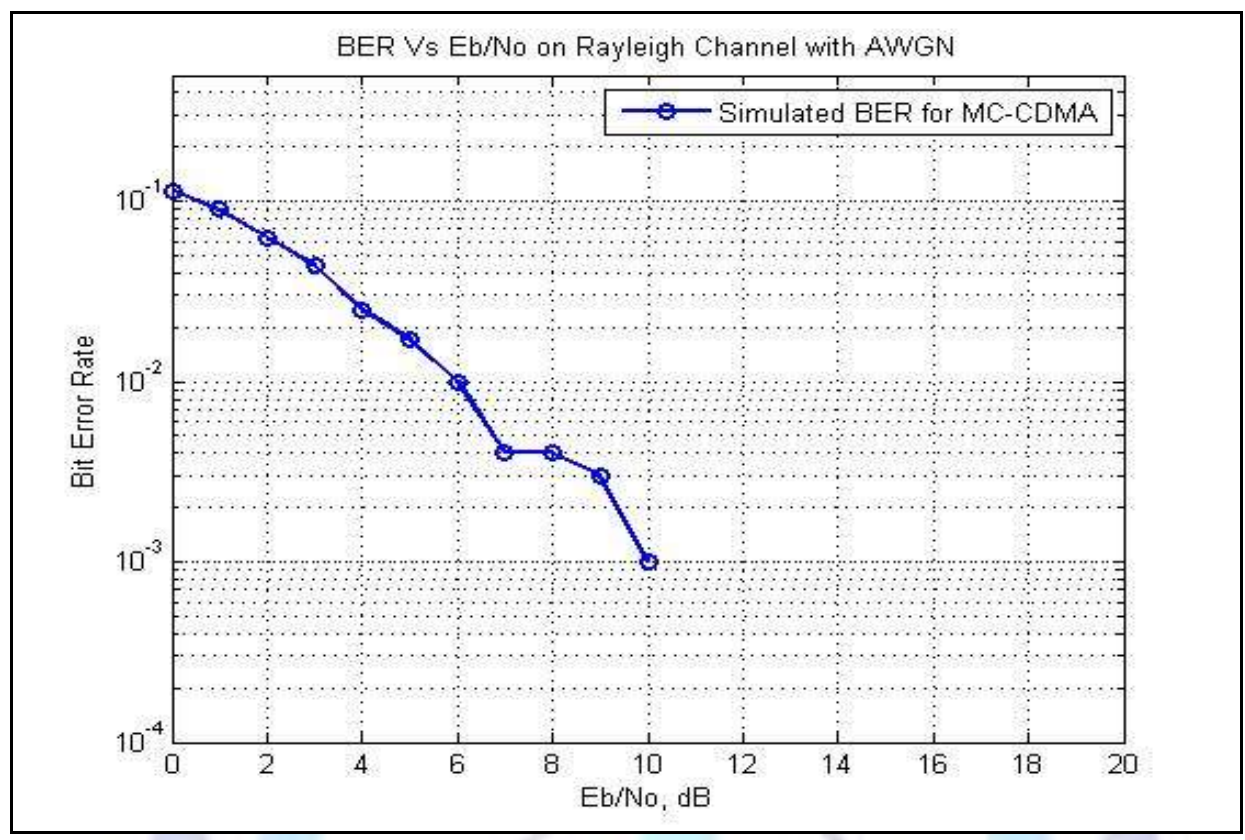

Figure 6: SNR/BER for 16 users 16 sub-carriers.

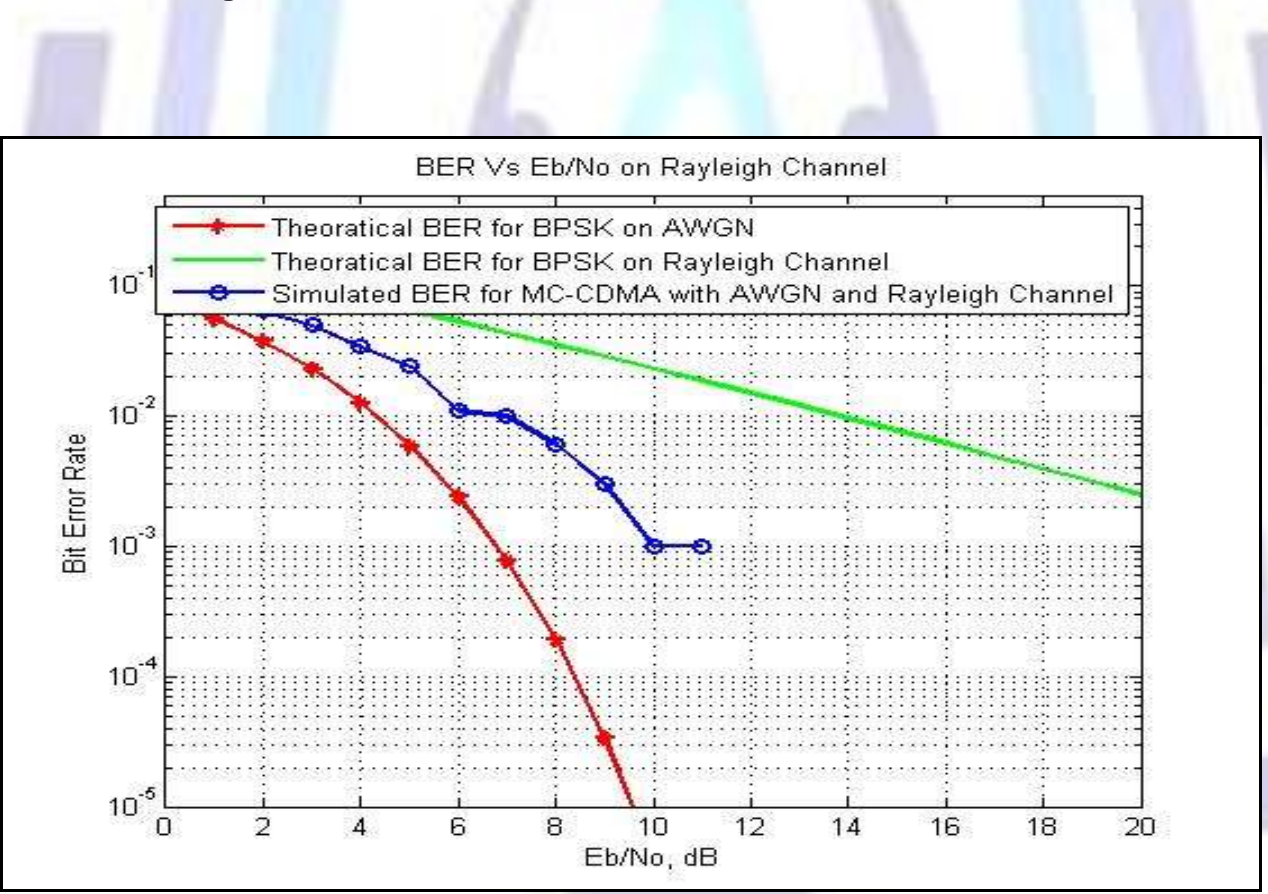

Figure 7: SNR/BER for 2 users 16 sub-carriers compared with BPSK system.

\section{CONCLUSION}

The MC-CDMA system for the AWGN with Rayleigh Fading Channel is simulated and also compared with the BPSK system and the simulation results shows that the MC-CDMA performs much better that the BPSK system under fading conditions. The simulation results also shows that the increase in the number of users with maintaining the number of sub carriers constant does not affects the system performance 


\section{REFERENCES}

http://en.wikipedia.org/wiki/Multicarrier_code_division_multiple_access.

[1] [2] Xue Li, Ruolin Zhou, Steven Hong and Zhiqiang Wu "Total Inter-Carrier Interference Cancellation for MC-CDMA System in Mobile Environment”, IEEE Globecom 2010 proceedings.

[2] [3] B.Sarala and D.S.Venkateswarulu "MC CDMA PAPR REDUCTION TECHNIQUES USING DISCRETE TRANSFORMS AND COMPANDING", International Journal of Distributed and Parallel Systems (IJDPS) Vol.2, No.6, November 2011

[3] [4]Georgios Orfanos, JorgHabethaand Ling Liu "MC-CDMA Based IEEE 802.11 Wireless LAN"

[4] [5] S.M.Navidpour, P. Amirshahi and M. Kavehrad "Performance Analysis of Coded MC-CDMA in Powerline Communication Channel with Implusive Noise", 2006 IEEE.

[5] [6] Kattoush A. H. and Qasaymeh M. M. "Design and Simulation of MC-CDMA Transceiver via Slantlet Transform", I.J. Information Technology and Computer Science, 2012, 3, 16-23.

[6] [7] "Channel Estimation for MIMO MC-CDMA Systems", International Journal of Distributed and Parallel Systems (IJDPS) Vol.2, No.6, November 2011.

\section{Author' biography}

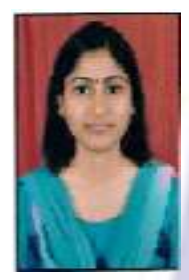

Bhavana kulhare, Received degree B.E in Electronics \&communication from R.G.P.V. Bhopal university \& M.TECH. in digital communication from BUIT,BU, Barkatullah University, Bhopal\& \& 6 years teaching experience 